\title{
aBETting therapeutic resistance by Wnt signaling
}

\author{
Cell Research (2015) 25:1187-1188. doi:10.1038/cr.2015.127; published online 30 October 2015
}

BET inhibition has emerged as a promising epigenetic therapy for malignancies in the last five years, but little consensus has developed regarding what may mediate the axis between sensitivity and resistance. Two recent papers published in $\mathrm{Na}$ ture attempt to address this question in acute myeloid leukemia (AML) and independently identify the Wnt signaling pathway as a driver and biomarker of therapeutic resistance.

In a recent issue of Nature, two reports investigate the mechanisms by which acute myeloid leukemia (AML) develops resistance to therapeutic inhibition of bromodomain and extra-terminal (BET) proteins. Small molecules in this class such as JQ1 and I-BET bind and inhibit the bromodomain modules - deep, hydrophobic pockets that recognize acetylated lysine residues on histones and transcription factors - to disrupt the productive transcription of key proliferative genes [1]. In particular, BET inhibition has emerged as one of the most promising strategies to target the potent oncogene $M Y C$ at the transcriptional level [2], although other action models have emerged including disruption of the binding of BRD4 to super-enhancers [3] or to transcription factors [4]. Now two groups, working from different angles, have evaluated a comprehensive set of models covering both intrinsic and acquired resistance to BET inhibition, and Wnt signaling emerged as a common theme and a key player mediating resistance to BET inhibitors.

Fong and colleagues [5] generated murine models of BET inhibitorresistant AML by serially passaging
MLL-AF9-transduced hematopoietic stem and progenitor cells (HSPCs) in increasing concentrations of I-BET. Resistant clones showed decreased sensitivity to I-BET in proliferation assays, accompanied by reduced apoptosis and limited cell cycle arrest. Mice grafted with these cells lost the survival advantage conferred by I-BET treatment. They also produced a similar model in vivo through serial transplantation of MLL-AF9 HSPCs in mice treated with I-BET.

The resistant cell lines generated by Fong et al. [5] showed a loss of myeloid lineage markers Gr1 and CD11b and an increase in tumor initiating capacity in limiting dilution assays in vivo. This raised the possibility that resistance to I-BET emerges from a leukemic stem cell (LSC) compartment. Indeed, I-BET-resistant populations showed enrichment for the LSC immunophenotype, and gene set enrichment analysis (GSEA) of RNA sequencing data revealed enrichment for a published LSC gene signature in I-BET-resistant cells. Sustained treatment with I-BET appeared to be necessary for the development and maintenance of the full resistance phenotype as I-BET-naive leukemic granulocyte macrophage progenitors were only modestly resistant to BET inhibition and withdrawal of I-BET from resistant cell lines led to an intermediate phenotype.

GSEA transcriptome analysis by Fong et al. [5] revealed upregulation of the Wnt/ $\beta$-catenin signaling pathway, which has also been implicated in cancer stem cell maintenance [6]. Importantly, they showed that inhibition of the Wnt pathway restored the more mature myeloid phenotype, survival advantage conferred by I-BET in resistant clones, and abrogation of $\mathrm{Myc}$ expression. Moreover, they showed an increased occupancy of BRD4 target genes by $\beta$-catenin in resistant cell lines both at regulatory elements of $M y c$ and globally, suggesting that in the absence of BRD4 activity, the $\mathrm{Wnt} / \beta$-catenin pathway is able to restore expression of genes critical for proliferation.

In a separate study that focused on intrinsic resistance, Rathert and colleagues [7] employed a screen against an shRNA library of 626 chromatin modifiers, aiming to identify crucial factors that confer BET inhibitor resistance to the murine MLL-AF9; $\mathrm{Nras}^{\mathrm{G} 12 \mathrm{D}}$ model of AML. Cells expressing shRNAs targeting Suz12, Psip12, and Dnmt3 showed robust resistance to JQ1 treatment. Interestingly, depletion of Suz12 and other polycomb repressive complex 2 (PRC2) components decreased cell proliferation in the absence of JQ1 treatment; however, when treated with JQ1, cells with such deficiency showed survival advantage in competitive proliferation assays, underscoring the highly context-dependent nature of PRC2's activity.

While Rathert et al. [7] did not find major differences in gene expression between sensitive and resistant clones upon short-term treatment with JQ1, resistant clones over time displayed divergent gene expression patterns including the restoration of multiple crucial transcripts such as Tifab and Myc. Rebound expression of $M y c$ was also a feature of resistant human AML cell lines versus sensitive lines despite initial loss in both. Interestingly, they found 


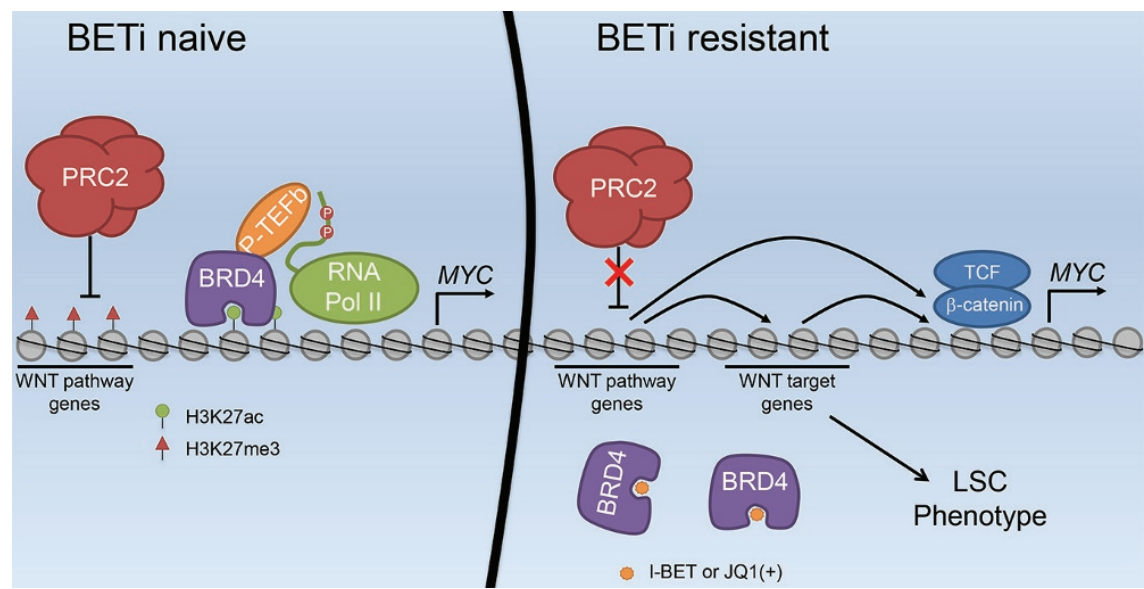

Figure 1 During normal cellular conditions, BRD4 recognizes acetylated lysine residues on histones and recruits positive transcription elongation factor $b(P-T E F b)$ to promote transcriptional elongation by RNA polymerase II of key oncogenic drivers such as MYC. BET inhibition (BETi) prevents acetyl lysine binding by the bromodomains on BRD4 and abrogates expression of MYC. In resistant cells, however, repression of the Wnt pathway by the polycomb repressive complex 2 (PRC2) is dampened. Together with its target genes, Wnt signaling is able to restore expression of genes such as MYC and promote resistance to BET inhibition.

that $\mathrm{H} 3 \mathrm{~K} 27 \mathrm{me} 3$ remained unchanged at BRD4 target sites by long-term JQ1 treatment. However, the H3K27me3 mark was enriched at genes in many pathways that were upregulated in resistant clones after BET inhibition. These data suggested that the role of PRC2 might not be to regulate BRD4 target genes, but to repress compensatory pathways; among them, the Wnt signaling pathway emerged as a potent mediator of JQ1 resistance in a GSEA comparing gene expression patterns in control and shRNA-generated JQ1resistant clones. Supporting this notion, many Wnt target genes were found to be consistently upregulated by JQ1 treatment among a panel of resistant human AML cell lines compared with sensitive lines, and stimulation of canonical Wnt signaling was able to confer a similar resistance phenotype.

It will be interesting to investigate whether Wnt signaling itself or its target genes are able to restore the expression of crucial genes such as $M y c$, and how BET inhibition modulates Wnt activity on chromatin. BRD4 has been proposed as a mediator of chromatin dynamics, and the study by Rathert et al. [7] described shifts in H3K27ac enrichment patterns around enhancers due to BET inhibition in resistant cells. Notably, a putative $M Y C$ enhancer element emerged in the first intron of $P V T 1$ (a long non-coding RNA $3^{\prime}$ of $M Y C$ ); this element displays strong enhancer activity and is enriched for $\mathrm{H} 3 \mathrm{~K} 27 \mathrm{ac}$ and TCF7L2.

Although Wnt signaling emerged as a common theme in both studies, sequencing data from both groups suggest that other pathways may be involved in mediating the resistance phenotype. TGF- $\beta$ showed significant enrichment as revealed by GSEA in both studies, but its potential contribution awaits further investigation. Additionally, it remains to be determined whether these resistance mechanisms operate beyond AML, especially in solid tumors, where the biology of BET inhibition has demonstrated strikingly different features [4, 8-10].

A better understanding of the resistance to BET inhibition is urgently needed. BET inhibitor compounds are currently being investigated in early clinical trials for a variety of cancers.
Given the recent momentum to deliver personalized therapies that take into consideration the molecular composition of individual patients' cancers, these reports begin to provide a basis for the development of biomarkers toward rational patient selection and opportunities for combination therapeutics to overcome resistance. A number of Wnt inhibitors that target various steps in the signaling pathway are under investigation in the clinical and preclinical settings [11]. As we gain a better understanding of how Wnt signaling is activated to promote resistance, we may be able to determine which Wnt inhibitors can buttress BET inhibition, rendering the therapy more robust and applicable to a broad patient base.

Carl G Engelke ${ }^{1,2}$, Arul M Chinnaiyan ${ }^{1,2,3,4,5}$

${ }^{I}$ Michigan Center for Translational Pathology, ${ }^{2}$ Department of Pathology, ${ }^{3}$ Department of Urology, University of Michigan, Ann Arbor, MI 48109, USA; ${ }^{4}$ Howard Hughes Medical Institute, University of Michigan Medical School, Ann Arbor, MI 48109, USA; ' Comprehensive Cancer Center, University of Michigan Medical School, Ann Arbor, MI 48109, USA

Correspondence: Arul M Chinnaiyan

E-mail: arul@umich.edu

\section{References}

1 Shi J, Vakoc CR. Mol Cell 2014; 54:728736.

2 Delmore JE, Issa GC, Lemieux ME, et al. Cell 2011; 146:904-917.

3 Lovén J, Hoke HA, Lin CY, et al. Cell 2013; 153:320-334.

4 Asangani IA, Dommeti VL, Wang X, et al. Nature 2014; 510:278-282.

5 Fong CY, Gilan O, Lam EYN, et al. Nature 2015; 525:538-542.

6 Holland JD, Klaus A, Garratt AN, et al. Curr Opin Cell Biol 2013; 25:254-264.

7 Rathert P, Roth M, Neumann T, et al. Nature 2015; 525:543-547.

8 Lockwood WW, Zejnullahu K, Bradner JE, et al. Proc Natl Acad Sci USA 2012; 109:19408-19413.

9 Nagarajan S, Hossan T, Alawi M, et al. Cell Rep 2014; 8:460-469.

10 Baker EK, Taylor S, Gupte A, et al. Sci Rep 2015; 5:10120.

11 Takebe N, Miele L, Harris PJ, et al. Nat Rev Clin Oncol 2015; 12:445-464. 